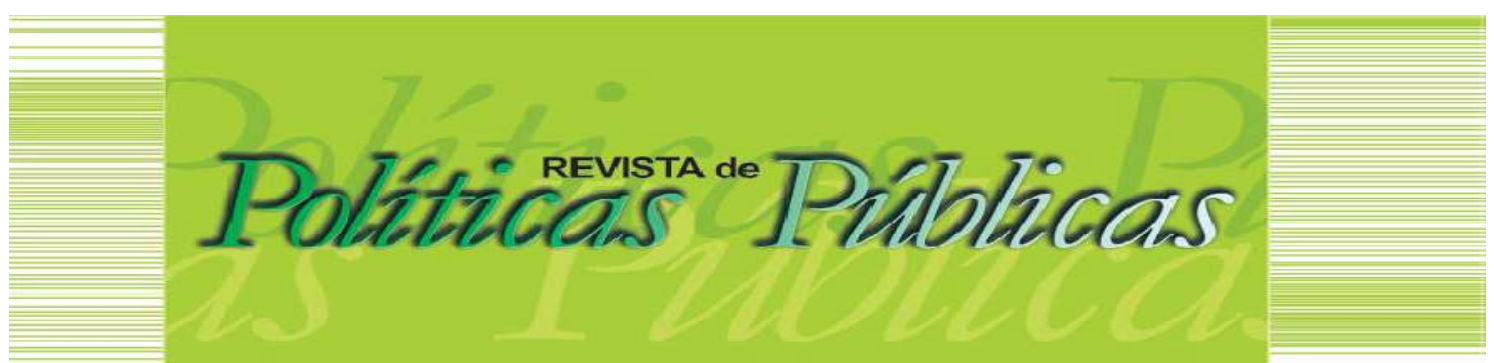

\title{
POLÍTICAS Y MODELOS DE INTERVENCIÓN LOCAL EN MATERIA SOCIOPRODUCTIVA Y AMBIENTAL: un análisis en el contexto argentino reciente
}

\author{
Rodrigo Reynaldo Carmona ${ }^{1}$
}

\section{Resumen}

El artículo busca examinar las distintas políticas y modelos de intervención local en materia socioproductiva y ambiental en tres municipios importantes de la Provincia de Buenos Aires (San Martín, Malvinas Argentinas y Tigre), en el período 2015.2018. Se presentan así los principales debates sobre políticas locales, relaciones intergubernamentales, gobernanza y desarrollo sostenible. Posteriormente, se analiza cada municipio considerando los principales programas y modelos de intervención local, con especial atención en las capacidades municipales vis a vis los niveles superiores de gobierno. En consecuencia, se pretende dar cuenta de cómo se configuran los distintos modelos de intervención local, los rasgos específicos que asumen las iniciativas desplegadas y los alcances políticos e institucionales desarrollados.

Palabras-claves: Políticas socioproductiva y ambiental. Municipios. Modelos de intervención local

POLICIES AND MODELS OF LOCAL INTERVENTION IN SOCIO-PRODUCTIVE AND ENVIRONMENTAL MATTERS: An analysis in the recent argentine context

\begin{abstract}
The article seeks to examine the different policies and models of local intervention in socio-productive and environmental matters in three important municipalities of the Province of Buenos Aires (San Martín, Malvinas Argentinas and Tigre), in the period 2015-2018. The main debates on local policies, intergovernmental relations, governance and sustainable development are presented. Subsequently, each municipality is analyzed, it evaluates the main programs and models of local intervention, with special attention on municipal capacities compared to higher levels of government. Consequently, it is expected to give an account of how the different models of local intervention are configured, the specific aspects assumed by the initiatives deployed and the political and institutional scope developed.
\end{abstract}

Keywords: Socio-productive and environmental policies. Municipalities. Local intervention models

Artigo recebido em: 16/12/2019 Aprovado em: 23/04/2020 DOl: http://dx.doi.org/10.18764/2178-2865.v24n1p190-204.

\footnotetext{
1 Licenciado en Ciencia Política, Universidad de Buenos Aires (UBA). Posdoctor en Ciencias Humanas y Sociales de la Facultad de Filosofía y Letras, Universidad de Buenos Aires (UBA). Doctor en Ciencias Sociales por la Facultad Latinoamericana de Ciencias Sociales, Mención Honorífica (FLACSO-Sede Argentina). Profesor e investigador asociado de las áreas "Estado, Gobierno y Administración Pública" y "Sistemas Económicos Urbanos" del Instituto del Conurbano (ICO) de la Universidad Nacional de General Sarmiento (UNGS) e investigador de carrera del Consejo Nacional de Investigaciones Científicas y Tecnológicas (CONICET) de Argentina. J.M. Gutierrez 1150 (B1613), Los Polvorines, Buenos Aires. E-mail: rcarmona@ungs.edu.ar.
} 


\section{INTRODUCCIÓN}

El presente trabajo tiene como objetivo central analizar las distintas políticas y modelos de intervención local en materia socioproductiva y ambiental en tres municipios importantes de la Provincia de Buenos Aires (San Martín, Malvinas Argentinas y Tigre), en el período 2015-.2018. En estos términos, el trabajo pretende dar cuenta de cómo se configura la matriz institucional y de políticas locales orientadas a estas problemáticas, las capacidades municipales vis a vis los niveles superiores de gobierno y qué desafíos en términos de coordinación intergubernamental se abren en esta nueva etapa.

El artículo se organiza del siguiente modo. En primer lugar, se presenta el marco conceptual dando cuenta de los principales aspectos que hacen a las políticas locales, las relaciones intergubernamentales, la innovación y desafíos de gobernanza en el escenario reciente. A continuación, se contemplan los aspectos metodológicos y las distintas tendencias y estrategias de desarrollo utilizadas en los municipios considerados, como el alcance de las políticas e intervenciones públicas desplegadas. Por último, se esbozan algunas conclusiones finales sobre las temáticas de referencia.

\section{POLÍTICAS LOCALES, RELACIONES INTERGUBERNAMENTALES, INNOVACIÓN Y GOBERNANZA}

Las reformas económicas de ajustes estructurales llevadas a cabo en América Latina en la década de los noventa, junto al proceso de descentralización y transferencia de funciones del nivel nacional hacia los niveles subnacionales, determinaron nuevas dinámicas en el plano territorial. Estas políticas para el caso argentino tuvieron un impacto de carácter doble sobre los gobiernos locales. En primer término, los municipios sufrieron una ampliación de sus funciones y responsabilidades y en menor medida de sus atribuciones, debiendo proveer un conjunto mayor y más heterogéneo de servicios a la comunidad, sin que ello implicara mayormente un incremento de recursos (técnicos, jurídicos, económicos, humanos y administrativos) acorde al crecimiento y complejidad de sus tareas. En segundo lugar, generaron un proceso de resignificación y en alguna medida jerarquización del ámbito local. Los municipios, en tanto primer "ventanilla" gubernamental, aparecieron revalorizados por su mayor grado de cercanía y receptividad para resolver problemas y conflictos ante los cambios en el nuevo escenario (CRAVACUORE, 2004; CABRERO MENDOZA, 2005; CARMONA, 2012).

Si bien los límites de lo "local" pueden cambiar en función de las características históricas y culturales y los procesos funcionales que adoptan los emplazamientos territoriales, es posible 
señalar a la órbita municipal como una instancia central de las diferentes interacciones sociales, económicas y político-institucionales. En consecuencia, uno de los ejes fundamentales a escala local/municipal se relaciona con las actividades productivas, el empleo y lo ambiental . La existencia de un modelo de intervención a nivel municipal sustentado en la articulación de los actores locales puede generar condiciones favorables para un desarrollo sustentable, si bien ello estará determinado por los estímulos y políticas desplegadas fuertemente desde el gobierno nacional y /provincial. En estos términos, el establecimiento de relaciones asociativas y de cooperación, entre empresas, organizaciones locales y el Estado constituyen aspectos importantes en la creación de un ambiente propicio que resulte en un aumento de la capacidad productiva y competitiva de estos espacios, junto al cuidado del medio ambiente (BOSCHERINI Y POMA, 2000; CAMAGNI, 2002; MAZORRA ET AL, 2005; PÉREZ, 2006: CARMONA, 2008).

En este escenario, las formas de hacer política y los estilos de gestión local han sido abordados en la literatura para el caso argentino. Distintos trabajos han enfatizado en las nuevas responsabilidades en materia social y económica que los gobiernos locales han debido asumir de "hecho" frente a las transformaciones estructurales, sin contar en la mayoría de los casos con capacidades y recursos necesarios para afrontar este tipo de acciones. La transferencia de la carga de la crisis por medio de los procesos de descentralización aumentó las funciones a ser ejercidas por los municipios. y obligó también a definir estrategias de legitimación del gobierno local para dar cuenta de la gestión del nuevo escenario (GARCÍA DELGADO, 1997; CHIARA, 1999; CRAVACUORE, 2007; DE PIERO, 2010; CARMONA, 2012; CASALIS, 2017).

Resulta relevante indagar así los principales instrumentos de política municipal y las relaciones intergubernamentales (RIG) establecidas. En primer lugar, es posible partir de la idea de matriz o modelo de intervención como el conjunto de políticas o iniciativas estatales implementadas a nivel municipal, más allá de que su diseño se origine en otra jurisdicción. En esta matriz intervienen actores estatales y no estatales en una dinámica definida por los recursos relativos que estos poseen y los marcos institucionales dentro y sobre los cuales estos operan (CARMONA Y ANZOÁTEGUI, 2010; COUTO, FOGLIA Y ROFMAN, 2016). Del mismo modo, consideramos a las RIG como un concepto amplio que remite a los vínculos entre las distintas unidades de gobierno existentes al interior de un Estado, esto es, un conjunto de actividades 0 interacciones que ocurren entre las unidades gubernamentales que integran el aparato estatal Por ende, las RIG son los mecanismos políticos y administrativos que colaboran en regular, organizar y, en última medida, viabilizar la gestión pública entre los diferentes niveles que integran un Estado (WRIGHT, 1997; MÉNDEZ, 1997; JORNADA, 2002; ESCOLAR et al, 2011). 
En estos términos, se destaca la relevancia que alcanza el concepto de innovación como forma de cambio institucional y organizacional. Se parte de pensar a la innovación como una construcción social compleja cuyo desarrollo supone una variedad de factores individuales, organizacionales y contextuales involucrados. La capacidad de las organizaciones para generar vínculos intraorganizacionales y con su entorno se transforma, así, en un aspecto central, de la misma forma que el origen del proceso innovador y sus particularidades en términos de amplitud y participación de los actores involucrados. Esto sugiere, por lo tanto, que la innovación, en tanto instancia abierta y construida, aparece como resultado de la capacidad organizacional interna en correspondencia con las posibilidades que brinda el contexto social y político (CRAVACUORE, ILARI Y VILLAR, 2004; CABRERO MENDOZA, 2012).

Estos aspectos, pensados en el ámbito local, subrayan la significación que asume la innovación como proceso integral que incluye tanto la dimensión administrativa o de la gestión como la política y contextual. La ampliación de las funciones tradicionales de los gobiernos locales se plantea entonces respecto a la apertura incipiente de sus agendas y el desarrollo de nuevos roles estratégicos e innovadores de mayor calidad en la intervención. En segundo lugar, se evidencia una dinámica de ampliación y extensión de la oferta de políticas públicas hacia otros planos (medio ambiente, desarrollo local, empleo y promoción económica, políticas socioculturales). Esto muestra que la definición tradicional y sectorial a nivel de políticas se combina en los últimos años con un aumento de una formulación más integral y horizontal de la gestión local, variable según los contextos particulares. Por otra parte, surgen nuevas estrategias y visiones globales respecto a los diversos procesos económicos, sociales y políticos que intervienen en la escena urbana. Distintas iniciativas de tipo transversal y estratégico se despliegan e inciden sobe los distintos campos de políticas públicas (BLANCO; SUBIRATS, 2009).

Las administraciones públicas son llamadas, entonces, a renovar sus formas de actuación, de modo de ser más flexibles y adaptables a escenarios sociales cada vez más cambiantes. Por ende, se torna necesario hablar cada vez más en términos de gobernanza, a nivel de mecanismos de articulación y coordinación entre diferentes actores. Los procesos sociopolíticos en espacios territoriales se establecen, así, a partir de una situación de interdependencia e influencia entre múltiples actores y niveles de intervención. Puede aseverarse, entonces, que la gobernanza en la literatura especializada plantea el pasaje gradual de una modalidad de actuación gubernamental sostenida en la primacía excluyente del gobernante en términos de la dirección y conducción política, a un escenario donde se establecen mayores grados de interacción y dependencias mutuas entre las instancias político-institucionales y sociales. De esta forma, resulta central la puesta en marcha de estrategias de desarrollo territorial sobre un eje de gobernanza, que permita estimular interacciones dinámicas entre 
los diversos actores intervinientes redes multinivel, "multiescalares" o multigeográficamente compuestas, que sean coherentes con el entorno y las trayectorias en donde se aplican (AGUILAR VILLANUEVA 2007; VIGIL Y FERNÁNDEZ, 2012).

\section{MUNICIPIOS CONSIDERADOS Y METODOLOGÍA DE ANÁLISIS}

Los municipios seleccionados para el análisis son San Martín, Malvinas Argentinas y Tigre , ubicados en el primer y segundo cordón del Conurbano Bonaerense dentro de la Provincia de Buenos Aires. En términos sociales y demográficos, los tres municipios cuentan con una cantidad de población importante, siendo San Martín el más densamente poblado con casi medio millón de habitantes en un territorio más pequeño que los otros distritos (en especial, Tigre, con una parte importante de su superficie compuesto por islas) (Cuadro 1).

Cuadro 1 - Indicadores sociodemográficos para los municipios seleccionados

\begin{tabular}{|c|c|c|c|}
\hline & San & Malvinas & Tigre \\
& Martín & Argentinas & \\
\hline Superficie (km2) & 57 & 63 & 368 \\
Población (2010) & 481.864 & 322.375 & 376.381 \\
Densidad & 8.418 & 5.117 & 1,022 \\
& & & \\
\hline
\end{tabular}

Fuente: Elaboración propia sobre la base de datos del INDEC (2010).

En particular, los tres casos para el estudio de las políticas poseen un perfil predominantemente industrial y concentran el $22.3 \%$ de la producción industrial del Conurbano Bonaerenses, siendo en especial General San Martín el que más peso alcanza en el total industrial con un $14.5 \%$ del producto. En relación a los niveles de Producto Bruto Geográfico (PBG) per cápita, General San Martín duplica a Tigre y triplica a Malvinas Argentinas. En términos de Necesidades Básicas Insatisfechas (NBI), que mide la pobreza estructural, General San Martín obtiene la mitad de los valores que los otros municipios (Cuadro 2).

Cuadro 2 - Producto Bruto Geográfico per cápita1, participación de la industria y cantidad de hogares con NB| ${ }^{2}$ para los municipios seleccionados y el conjunto del Conurbano Bonaerense

\begin{tabular}{|l|c|c|c|}
\hline Municipios & $\begin{array}{c}\text { PBG } \\
\text { per } \\
\text { cápita } \\
\mathbf{( 2 0 0 8 )}\end{array}$ & $\begin{array}{c}\text { Participación } \\
\text { de la Industria } \\
\text { en el } \\
\text { Conurbano } \\
\text { Bonaerense \% }\end{array}$ & $\begin{array}{c}\text { Hogares } \\
\text { por NBI } \\
\mathbf{( 2 0 1 0 )} \\
\text { \% }\end{array}$ \\
\hline General San Martín & 38.913 & 14.5 & 7.6 \\
\hline Tigre & 19.077 & 4.8 & 13 \\
\hline Malvinas Argentinas & 13.857 & 3 & 14 \\
\hline Conurbano Bonaerense & 19.001 & 68,2 & 10,8 \\
\hline
\end{tabular}

Fuente: Elaboración propia en base a Lodola et al (2010) y Censo Nacional de Población 2010. 
Los casos seleccionados son municipios gobernados por dos de las tres principales fuerzas político partidarias a nivel regional y nacional: Frente para la Victoria-Partido Justicialista (dos casos) y Frente Renovador (un caso). De este modo, se busca indagar a partir del nuevo mapa político que se abre a finales del año $2015^{3}$, cómo incide el cambio de contexto macroeconómico y político en la configuración productiva y qué desafíos en términos de gobernanza se abren a partir del nuevo escenario.

De esta forma, para el trabajo, se propone una metodología cualitativa de investigación buscando examinar cómo se configuran los modelos de intervención en cada uno de los municipios y la dinámica de actores e instituciones en los diferentes ámbitos. Para ello, se analiza el grado de autonomía financiera municipal (la relación entre los recursos corrientes municipales versus los provenientes de otras jurisdicciones), el perfil político del partido gobernando y las políticas e iniciativas desplegadas en cada caso. Ello configura un modelo de intervención local que permite una aproximación a las redes de gobernanza establecidas, como así también indagar las políticas en materia socioproductiva y ambiental desplegadas. Para ello se recopila información desagregada para los tres casos municipales seleccionados.

En este contexto, el modelo de intervención municipal en materia socioproductiva y ambiental puede definirse a partir de la siguiente operacionalización que se detalla a continuación. Por un lado, se pondera 1) la extensión del modelo, según el número de iniciativas implementadas en el territorio. Asimismo se analiza 2) la diversificación del modelo, según los sujetos destinatarios de las políticas, considerándose "especializado" o "diversificado", según la cantidad de sujetos destinatarios. Por otro lado, se evalúa 3) la autonomía municipal para desplegar iniciativas propias, según la cantidad de iniciativas municipales vis a vis las provenientes de otras jurisdicciones, siendo el modelo más "autónomo" o dependiente "dependiente" cuando predominan las iniciativas provenientes de Nación o Provincia. Finalmente, 4) la institucionalización refiere a la jerarquización y envergadura de las estructuras municipales dedicadas a las áreas consideradas, siendo "alta" o "baja", según si el rango alcanza el nivel de Subsecretaría y la misma contiene los programas identificados en la materia.

El modelo de intervención designa así al conjunto de políticas, programas e iniciativas, provenientes de diferentes niveles jurisdiccionales (Nación, Provincia, Municipios), que involucran a actores públicos y privados locales y se implementan en el ámbito municipal. El término permite una aproximación a la extensión de la intervención estatal municipal en el territorio, a la diversidad de actores involucrados e interpelados por las políticas, así como a las capacidades y autonomía municipales para desplegar iniciativas propias e implementar instrumentos que involucran diversas competencias técnicas. Asimismo, la reconstrucción del modelo permite ponderar el peso de las iniciativas propias vis a vis las provenientes de otras jurisdicciones, caracterizando la relación 
intergubernamental. La elaboración del modelo se nutre de información primaria proveniente de entrevistas semiestructuradas a referentes y funcionarios de las áreas de producción, industria y empleo, de información proveniente de sitios web municipales y bibliografía secundaria.

\section{MODELOS DE INTERVENCIÓN LOCAL}

La gestión de los municipios considerados presenta, en base al escenario expuesto, grandes desafíos para promover un desarrollo sostenible. La elevada densidad poblacional y la concentración económica ejercen fuerte presión sobre el mercado de trabajo, sobre la oferta de servicios públicos y a la vez demandan de los gobiernos locales un grado significativo de innovación en materia de planificación urbana y ordenamiento territorial. Como primer interlocutor, los gobiernos locales son interpelados por diferentes colectivos y actores sociales y económicos en torno a temáticas diversas.

En particular la cuestión socioproductiva y ambiental han adquirido protagonismo en las agendas locales, corriendo los límites de la agenda tradicional municipal argentina.

acondicionamiento del entorno productivo entrañaba incumbencias ligadas a la realización de obras públicas, la prestación de servicios públicos (agua, redes cloacales, alumbrado, etc.) y la habilitación, seguridad y regulación de los emprendimientos productivos.

En grados diversos las funciones municipales se ampliaron en torno a una agenda de desarrollo local, de puesta en valor de los entornos de producción, de despliegue de capacidades y competencias de los actores para el mercado a través de vinculaciones con el sistema educativo, y también con el cuidado del medioambiente. Por otra parte, la creciente desigualdad socioeconómica territorial derivó en nuevas funciones de contención y resolución de problemas sociales desde los gobiernos locales vinculados a la salud, la educación y también la problemática laboral.

En este marco, al proceso de descentralización de los años noventa y revitalización de los gobiernos locales, le siguió una etapa entre 2003 y 2015 de fuertes transferencias de recursos desde el nivel nacional y provincial al local, que marcó una creciente agenda e intervención sobre el territorio en materia social y económica. El incremento de recursos hacia los municipios fue significativo y combinó transferencias desde la provincia y la nación mayormente, en un proceso de competencia interjurisdiccional por el control político sobre el territorio. Gran parte de los recursos transferidos estuvieron ligados a obras de infraestructura, a programas e instrumentos socioproductivos de desarrollo local que interpelaron a los municipios y demandaron desplegar importantes capacidades de gestión, en muchos casos que no se habían desarrollado antes. 
Si bien dicha expansión de funciones se materializa, se observa una gran inestabilidad de las estructuras estatales en el plano local. Incluso en municipios con continuidad de la gestión político partidaria se registra gran rotación de funcionarios en diferentes áreas, expansión y contracción de las dependencias y los instrumentos de intervención. Y en los casos que experimentan cambios del signo político, los ajustes de estructuras y procesos municipales son más profundos.

Desde fines del año 2015 hasta la actualidad, se fue estableciendo desde el gobierno nacional un esquema de intereses y políticas de orientación neoliberal. Distintas intervenciones públicas tuvieron como objeto generar un importante "cambio" de rumbo respecto a la etapa anterior. El impacto de estos cambios a nivel local y en materia productiva se dio, por un lado, en la disminución de partidas transferidas desde Nación, destinadas fundamentalmente a programas sociales y socioproductivos (Argentina Trabaja, Programa de mejoramiento barrial, Mejor Vivir), para los cuales se destinaban transferencias significativas, y, por otro lado, el impacto operó a través de la nueva configuración política del Conurbano Bonaerense que a partir de las elecciones del año 2015 dejó de ser un territorio casi exclusivo del Partido Justicialista. En el nuevo escenario, la injerencia de los gobiernos locales para desplegar políticas e iniciativas en materia socioproductiva se ve condicionada. En materia ambiental, la problemática toma relieve como cuestión a ser considerada con algunas iniciativas municipales.

El análisis de los modelos de intervención en materia socioproductiva y ambiental, evidencia la presencia de un modelo extendido e institucionalizado en los tres municipios seleccionados no obstante se observan diferencias entre ellos (cuadro 3). De los casos considerados, Malvinas Argentinas presenta el modelo menos amplio en cuanto a la cantidad de iniciativas desplegadas. Asimismo, presenta el caso más dependiente, en cuanto a que sólo despliega una iniciativa localmente (Censo Industrial), frente a los restantes programas incorporados desde otras jurisdicciones. 
POLÍTICAS Y MODELOS DE INTERVENCIÓN LOCAL EN MATERIA SOCIOPRODUCTIVA Y AMBIENTAL: un análisis en el contexto argentino reciente

Cuadro 3 - Rasgos político-institucionales, financieros y modelos de intervención municipal

\begin{tabular}{|c|c|c|c|}
\hline $\begin{array}{c}\text { Municipio } \\
\text { Partido Gobernante } \\
\text { Autonomía Financiera } \\
\text { Antigüedad de la gestión } \\
\text { actual }\end{array}$ & $\begin{array}{l}\text { Modelo de intervención } \\
\text { municipal }\end{array}$ & $\begin{array}{l}\text { Principales políticas e iniciativas } \\
\text { (jurisdicción) }\end{array}$ & $\begin{array}{l}\text { Institucionalización/ } \\
\text { Funciones }\end{array}$ \\
\hline $\begin{array}{l}\text { Malvinas Argentinas } \\
\text { Frente p/la Victoria- Partido } \\
\text { Justicialista } \\
43,43 \% \\
2015-2019\end{array}$ & $\begin{array}{l}\text { Extendido, dependiente y diversificado. } \\
\text { Fuerte articulación con actores } \\
\text { industriales de la zona del Parque } \\
\text { Industrial/Triángulo }\end{array}$ & $\begin{array}{l}\text { Censo industrial municipal (Municipal) } \\
\text { Clínicas Tecnológicas (Provincia) } \\
\text { Recuperación. Productiva. (Nación) } \\
\text { Reconversión Productiva (Nación) } \\
\text { Jóvenes (Nación) } \\
\text { Programa Reciclado de Residuos } \\
\text { Sólidos Urbanos (Municipalidad) }\end{array}$ & $\begin{array}{l}\text { Alta. Secretaria de } \\
\text { Producción Industria y } \\
\text { Medioambiente. } \\
\text { Dir. de Economía Social }\end{array}$ \\
\hline $\begin{array}{l}\text { General San Martín } \\
\text { Frente p/ la Victoria- } \\
\text { Partido Justicialista } \\
63,49 \% \\
2011-2019\end{array}$ & $\begin{array}{l}\text { Extendido, autónomo y diversificado. } \\
\text { Fuerte despliegue de iniciativas y } \\
\text { programas de desarrollo productivo } \\
\text { general, economía social y empleo. }\end{array}$ & $\begin{array}{l}\text { Plan } 111 \text { Mil (Nación) } \\
\text { UNSAM } 1992 \text { (Nación) } \\
\text { San Martín Compra (Municipal), } \\
\text { Observatorio Socioeconómico } \\
\text { (Municipal) } \\
\text { Programa de Asistencias. Técnica y } \\
\text { Ases (Municipal) } \\
\text { Parque industrial San Martín } \\
\text { (Municipal) } \\
\text { Programa RSE (Municipal) } \\
\text { Programa de Desarrollo Económico } \\
\text { Territorial (Municipal) } \\
\text { Programa Día Verde (Municipal) } \\
\text { Programa Separar, Reciclar, Crecer } \\
\text { (Municipal) }\end{array}$ & $\begin{array}{l}\text { Alta. Secretaría . de } \\
\text { Producción y. } 2 \\
\text { Subsecretarías (11 } \\
\text { Direcciones) + Agencia de } \\
\text { empleo multiactoral }\end{array}$ \\
\hline $\begin{array}{l}\text { Tigre } \\
\text { Frente Renovador } \\
73,67 \% \\
2013-2019\end{array}$ & $\begin{array}{l}\text { Extendido, autónomo y diversificado en } \\
\text { iniciativas y programas de desarrollo } \\
\text { productivo emprendedor, economía } \\
\text { social y empleo. }\end{array}$ & $\begin{array}{l}\text { UTN (Nación), Jóvenes con Más } \\
\text { Empleo (Nación) } \\
\text { PROMOVER (Nación) } \\
\text { Programa Inserción Laboral (Nación) } \\
\text { Entrenamientos Laborales (Nación) } \\
\text { Asistencia .al emprendedorismo y } \\
\text { asociativismo (Municipal) } \\
\text { Capacit. Polo Tecno. Social } \\
\text { (Municipal) } \\
\text { Tarjeta Soy Tigre (Municipal) } \\
\text { Centro Universitario Tigre (Municipal) } \\
\text { Programa SEPARÁ (Municipal) } \\
\text { Plan del Manejo del Delta (Municipal) } \\
\text { Participación COMIREC (Ente } \\
\text { autárquico) }\end{array}$ & $\begin{array}{l}\text { Alta. Subsecretaría de } \\
\text { Empleo y Producción ( } 3 \\
\text { Direcciones) }\end{array}$ \\
\hline
\end{tabular}

Fuente: Elaboración propia

Malvinas Argentinas presenta también el grado más bajo de autonomía financiera y la antigüedad de gestión más breve, lo que se condiciona probablemente un modelo más dependiente, por la cantidad de iniciativas propias desplegadas (2 de 5). La gestión ha sido no obstante muy activa en las acciones de regularización productiva del municipio y en desarrollar herramientas de diagnóstico que le permitieran tener un mapa ajustado y actualizado de la realidad productiva industrial lo que llevó en 2018-2019 a implementar el Censo Industrial en articulación con la Universidad Nacional de General Sarmiento, emplazada en su distrito, y el INDEC. Finalmente, el municipio desarrolla una gestión diversificada en cuanto a los interlocutores que interpela a través de sus políticas, fuertemente orientada a los actores de la trama industrial y a la cooperativización de empresas en situación de 
crisis, como así también a los actores del asociativismo, a través de la Dirección de Promoción de la Economía Social (emprendedores, empresas recuperadas, medianos productores y artesanos). En ese marco, el tema ambiental respecto al tratamiento en origen de residuos y su vinculación con la industria resulta relevante para el municipio, desarrollando así un programa de reciclado de residuos sólidos urbanos en una planta de tratamiento propia.

En General San Martín, la autonomía financiera municipal es más elevada, con un modelo más expansivo que el anterior que comprende ocho iniciativas, algunas de gran envergadura como San Martín Compra o el Observatorio Socioeconómico. Asimismo, despliega gran número de iniciativas propias (8 de 10) y también provenientes de otras jurisdicciones, de modo que el modelo se despliega autónomamente, con gran capacidad de incorporación de herramientas de Nación o Provincia. La Universidad Nacional de San Martín asimismo, constituye una institución de gran envergadura que convoca iniciativas de desarrollo productivo y tecnológico de avanzada, con fuerte articulación con el entorno y la municipalidad. Por último, San Martín interpela una gran variedad de actores del entorno productivo general, de la economía social y el empleo formal. A nivel ambiental, el municipio despliega fundamentalmente actividades de concientización y cuidado sobre el medio ambiente y el reciclado de residuos

El caso de Tigre también es paradigmático de un modelo de intervención municipal extendido e institucionalizado con gran desagregación de la estructura municipal que atiende las diferentes funciones del desarrollo productivo. En esta materia, presenta 9 iniciativas, siendo 3 de gran envergadura. El Polo Tecnológico Social y el Centro Universitario Tigre involucran la movilización de importantes recursos destinados a la formación y capacitación orientados a la mejora de la empleabilidad local. Asimismo, Tigre posee en su territorio a la Sede Pacheco de la Universidad Tecnológica Nacional, de gran envergadura académica en áreas técnicas. En relación a lo ambiental, desde el municipio se desarrolla una activa agenda con diversas acciones: Plan del Manejo del Delta (control del ecosistema y reforestación -con la plantación de 400 árboles-), el Programa SEPARÁ (reciclado y tratamiento), el reclamo de saneamiento del Río Reconquista y su participación en el Comité de Cuenca del Río Reconquista (COMIREC)4. Del total de iniciativas, Tigre despliega un modelo autónomo (6 iniciativas propias de 12) y diversificado a un amplio abanico de actores: emprendedores, actores de la economía social y vinculados al empleo formal, ciudadanos en general.

En materia de articulación interjurisdiccional, dos de los tres casos presentan modelos más autónomos, es decir que las iniciativas municipales superan a las incorporadas desde otras jurisdicciones. Cabe mencionar que muchas de las políticas del gobierno nacional y provincial destinadas a la producción y el empleo en el período 2016-2017 son heredadas de la gestión anterior (Programa de Recuperación Productiva, Programa de Empleo Joven) y otras son nuevas, en especial 
las orientadas a la asistencia técnica y el desarrollo emprendedor (Clínicas Tecnológicas y un conjunto de iniciativas para emprendedores). Finalmente, en el año 2018 en el marco de un contexto de fuerte ajuste presupuestario en ambas jurisdicciones, los programas de Nación o de la Provincia se han acotado y han permanecido en muy pocos municipios. De los casos analizados sólo Tigre realiza un aprovechamiento amplio de la articulación con Nación para la implementación de programas de promoción del empleo. Por último, dos de los tres municipios ensayan iniciativas de compre local promoviendo facilidades de crédito a través de tarjetas locales (Tigre) 0 aprovechando el poder de compra pública municipal y desarrollo de proveedores (San Martín).

En relación con los actores de la trama socioproductiva, en los tres municipios el modelo de intervención es diversificado en la medida que se despliegan iniciativas que interpelan y benefician a un conjunto amplio de actores, emprendedores o trabajadores autónomos, desocupados, cooperativas o iniciativas asociativas y pymes. No obstante, Malvinas Argentinas y San Martín son más activos en articular programas orientados a empresas o cámaras empresarias en torno a proyectos asociativos, clusters o Parques Industriales. Estas iniciativas que hasta 2011 eran promovidas desde la Provincia a través del Programa Distritos Industriales, se discontinuó y quedó confinada a las líneas promocionales de Nación de Parques Industriales que tuvo su auge entre 2011 y 2015.

En cuanto a la producción local de información primaria, dos de los tres municipios poseen iniciativas de relevamiento de datos municipales en materia económica (Malvinas Argentinas y General San Martín). Existe una vacancia significativa de información pública oficial de Nación y Provincia desagregada a nivel municipal que complejiza la disponibilidad de datos necesarios para la elaboración de políticas locales, de modo que la creación de observatorios o encuestas propias desarrolladas por las áreas municipales 0 en articulación con las Universidades nacionales de la zona resultan innovaciones esenciales si el municipio se propone una sintonía fina en materia de intervención productiva.

Por último, respecto a la problemática ambiental la cuestión ha ganado fuerte protagonismo en el último tiempo en cada uno de los municipios. La temática del reciclado y tratamiento de residuos sólidos urbanos aparece como problemática relevante (con sus primeros avances y a los efectos de mitigar los efectos generales no solucionados), además de la concientización sobre el medio ambiente y, en un área de islas como la de Tigre, la cuestión del cuidado del ecosistema. Sin embargo, persisten cuestiones no saldadas como una mirada de conjunto para el aglomerado urbano respecto a la disposición final de residuos ante el relleno sanitario colapsado, la contaminación y el riesgo hídrico fundamentalmente en las cuencas. 


\section{REFLEXIONES FINALES}

El presente trabajo buscó examinar en los municipios considerados. las distintas políticas y modelos de intervención local en materia socioproductiva y ambiental. De este modo, se propuso trabajar sobre un enfoque alternativo que pudiera examinar el alcance de las políticas y las capacidades municipales vis a vis los niveles superiores de gobierno.

Se contempló, así, el grado de autonomía financiera municipal -en tanto relación entre los recursos corrientes municipales y los derivados de otras jurisdicciones-, el perfil político del partido gobernando y las políticas e iniciativas desplegadas en cada caso. La conformación y caracterización de un modelo de intervención local (a nivel socioproductivo y ambiental) permite apreciar las redes de gobernanza constituidas, como así también al desarrollo local sobre la base de las políticas de promoción y contención generadas.

En este sentido, se apuntó a indagar el conjunto de políticas, programas e iniciativas, resultantes de diferentes niveles jurisdiccionales (Nación, Provincia, Municipios), que comprenden a actores públicos y privados locales y se implementan en el ámbito municipal. Se buscó, así, dar cuenta de la intervención estatal en el territorio, la posibilidad de contemplar la multiplicidad de actores involucrados por las políticas, al mismo tiempo, que vislumbrar las capacidades y grados de autonomía municipal para desplegar iniciativas propias e implementar instrumentos. En consecuencia, el modelo de intervención local permite examinar el peso de las iniciativas propias vis a vis las provenientes de otras jurisdicciones, definiendo la relación intergubernamental.

De esta forma, la selección de tres casos municipales con fuerte incidencia de la industria en el producto (concentran el $22.3 \%$ de la producción del Conurbano Bonaerenses), permitió indagar con mayor énfasis los modelos de intervención que despliegan los gobiernos locales en materia socioproductiva y ambiental. Su operacionalización a través de cuatro elementos (1. extensión; 2. diversificación; 3. autonomía; 4. institucionalización), contribuyó a dar algunas conclusiones preliminares.

Los municipios considerados presentan modelos extendidos, evidencian un "alto" grado de institucionalización, mientras que la antigüedad de la gestión incide en los aprendizajes y acervos acumulados en el tiempo y en la continuidad de los equipos técnicos en las estructuras municipales. En especial ese acervo es menor en las estructuras que de los municipios que transitaron recambio de signo político partidario como Malvinas Argentinas, hacia el año 2015. Más específicamente y en comparación con los otros casos, éste último exhibe el modelo menos extendido en cuanto a la cantidad de iniciativas emprendidas y es más dependiente y menos autónomo respecto de los programas desplegados desde otras jurisdicciones. 
Asimismo, se observó que el perfil político-partidario de los tres casos seleccionados encierra un conjunto de premisas de intervención municipal bien activa. Las dos fuerzas políticas, el FPV-PJ y el FR-PJ son dos vertientes del Justicialismo o peronismo, que tradicionalmente posee agendas más intervencionistas con una lógica expansiva de iniciativas orientadas a la producción y el empleo, siendo la cuestión ambiental menos preponderante. Por otra parte, cabe señalar que en los tres municipios los esquemas de gestión trasvasan las funciones tradicionales de los municipios, ligadas a la provisión de servicios públicos básicos y de infraestructura urbana, y despliegan importantes acciones para atender los diversos problemas que acontecen en la actualidad.

Se evidencian así en líneas generales para los distintos casos intercambios y dinámicas desde una perspectiva de gobernanza multi-escalar, tanto a nivel vertical como horizontal. En un plano vertical, el gobierno local se distingue por ser el origen o destino respecto a las políticas públicas que se establecen en otros niveles jurisdiccionales. En el plano horizontal, busca dar apertura y curso a diversas demandas planteadas, generando iniciativas y programas locales propios. En un contexto complejo a nivel social y económico como el que caracteriza a los tres municipios, su impulso seguramente deberá estar determinado por una intervención coordinada y articulada entre los diferentes niveles gubernamentales.

En ese sentido, un esquema de análisis desde un enfoque de gobernanza que contemple el alcance de las políticas, las capacidades institucionales generadas y la articulación con los diversos actores y niveles de gobierno, resulta relevante para dar cuenta de las dinámicas de desarrollo presentes en el territorio y sus condiciones de partida. Indagar más en profundidad, los modelos de intervención y sus diversas implicancias locales, aparece como un desafío de suma importancia para pensar nuevas iniciativas públicas e institucionales con mayor grado de efectividad y legitimidad en términos de resultados a ser alcanzados en materia socioproductiva y ambiental.

\section{REFERENCIAS}

AGUILAR VILLANUEVA, Luis. El aporte de la Política Pública y de la Nueva Gestión Pública a la gobernanza. Revista del CLAD Reforma y Democracia, no 39, 2007.

BLANCO, Ismael, \& SUBIRATS, Joan ¿Existen territorios socialmente excluyentes? Contra lo inexorable. Respuestas locales a inseguridades globales. Innovación y cambios en Brasil y España, 119. 2009.

BOSCHERINIi, Fabio; POMA, Lucio. Más allá de los distritos industriales: el nuevo concepto de territorio en el marco de la economía global. En BOSCHERINli, Fabio; POMA, Lucio (comp.) Territorio, conocimiento y competitividad de las empresas, Buenos Aires, Miño y Dávila Editores, 2000. 
CABRERO MENDOZA, Enrique. Acción pública y desarrollo local. Fondo de Cultura Económica, 2012.

CAMAGNI, Roberto. On the concept of territorial competitiveness: sound or misleading?. Urban Studies, 39(13), 2395-2411, 2002.

CARMONA, Rodrigo El debate sobre nuevos estilos de gobierno en ciudades argentinas. CICCUS-UNGS, Bs. As, 2012.

CARMONA, Rodrigo. Repensando las formas de gobierno y gestión pública en grandes ciudades de la Argentina. Análisis en términos de políticas de articulación multiactoral y gobernanza democrática. En: CÓRDOVA MONTÚFAR, Marco. (coord) Lo urbano en su complejidad. Una lectura desde América Latina, Congreso Latinoamericano y Caribeño de Ciencias Sociales, Serie 50 años, FLACSO, Quito, Ecuador, 2008.

CARMONA, Rodrigo y ANZOÁTEGUI, Mirtha. Aspectos político-institucionales, modelos de gestión pública y tipo de articulación Estado-sociedad en municipios del oeste y noroeste del Conurbano Bonaerense. En ROFMAN, Adirana (comp.). Sociedad y territorio en el Conurbano Bonaerense. Un estudio sobre las condiciones socioeconómicas y sociopolíticas de cuatro partidos: San Miguel, José C. Paz, Moreno y Morón. Buenos Aires: UNGS, 2010.

CASALIS, Alejandro Procesos, dinámicas y tendencias de las políticas públicas nacionalesenrelación al desarrollo territorial y la gestión local en Argentina (2003-2017). En Divulgatio, Perfiles académicos de posgrado, Secretaría de Posgrado de la Universidad Nacional de Quilmes. Edición $N^{0} 3$, área temática: Economía, 2017.

CRAVACUORE, Daniel . La recentralización emergente en América Latina. En Fidel, Carlos; Villar, Alejandro (comps.). Miradas y Controversias del Desarrollo Territorial. Aproximación a un Enfoque Analítico. Buenos Aires: Ediciones del Centro Cultural de la Cooperación - Universidad Nacional de Quilmes, 2014.

CRAVACUORE, Daniel. Los municipios argentinos (1990-2005). En: CRAVACUORE, Daniel.; ISRAEL, Ricardo (Comps.). Procesos políticos municipales comparados en Argentina y Chile (1990-2005). Buenos Aires: Editorial de la Universidad Nacional de Quilmes-Universidad Autónoma de Chile, 2007.

CRAVACUORE, Daniel.; ILARI, Sergio y VILLAR, Alejandro La articulación de la gestión municipal. Actores y políticas. Buenos Aires, Universidad Nacional de Quilmes, 2004.

COUTO, Bárbara; FOGLIA, Carolina; ROFMAN, Adriana. Políticas participativas locales en municipios bonaerenses: una aproximación político-territorial. En ROFMAN, Adriana (comp.) Participación, Políticas Públicas y Territorio, Ediciones UNGS, 2016.

CHIARA, Magdalena. El nivel local de implementación de las políticas sociales: características y problemas. Algunas reflexiones en torno a los municipios del conurbano bonaerense", Ponencia presentada en la Jornada Pobres, Pobreza y Exclusión Social, UBA/CEILCONICET, Buenos Aires, 25 al 27 de agosto, 1999.

DE PIERO, Sergio Participación Ciudadana en Políticas Públicas. En: Recorridos del Tercer Sector. Informe de Desarrollo Humano de la Provincia de Buenos Aires (2009-2010) (pp. 64-7). La Plata: Fundación BAPRO, 2010. 
ESCOLAR, Marcelo; GONZÁLEZ, Lucas; CAMINOTTI, Mariana; QUILICCI, Federico; DEL COGLIANO, Natalia. Federalismo y Relaciones Intergubernamentales. Fragmentación política y federalismo ejecutivo: el caso argentino». X Congreso Nacional de Ciencia Política SAAP. Córdoba, 2011.

GARCÍA DELGADO, Daniel. Nuevos escenarios locales. El cambio de modelo de gestión. en GARCÍA DELGADO, Daniel (comp.) Hacia un nuevo modelo de gestión local. Municipio y sociedad civil en Argentina, Buenos Aires: FLACSO/UBA-CBC/Universidad Católica de Córdoba, 1997.

JORDANA, Jacint Relaciones Intergubernamentales y descentralización en América Latina. Casos de Argentina y Bolivia. Serie Documentos de Trabajo I-38 UE. Social InstituteDevelopment INDES-BID, 2002.

LODOLA, Agustín; BRIGO, Rafael y MORRA, Fernando. Economía de los Gobiernos Municipales. Teoría y Aplicaciones a la Argentina. Producción en los Municipios de la Provincia de Buenos Aires, Proyecto PICT 799/2007, Abril, 2010.

MAZZORRA, Ximena; FILIPPO, Agustín.; SCHLESSER, Diego. Áreas económicas locales y mercado de trabajo: estudios de tres casos, Serie Desarrollo productivo, No 157 (LC/L.2151-P), Santiago de Chile, Comisión Económica para América Latina y el Caribe (Cepal). Publicación de las Naciones Unidas, Nº de venta: S.04.II.G.79, 2005.

MÉNDEZ, José Luis. Estudio Introductorio. En: WRIGHT, Deil Para entender las relaciones intergubernamentales. México: Fondo de Cultura, 1997.

VIGIL, José Luis Y FERNÁNDEZ, Víctor Ramiro Gobernanza y regiones en perspectiva crítica: un abordaje para la construcción de políticas públicas, Revista del CLAD Reforma y Democracia. No. 53. (Jun. 2012). Caracas.

WRIGHT, Deil. Para entender las relaciones intergubernamentales. México DF: Fondo de Cultura Económica, 1997.

\section{Notas}

1 El Producto Bruto Geográfico per cápita resulta de dividir el Valor Agregado Bruto (VAB) entre la cantidad de habitantes de cada partido. Se optó por utilizar la población 2010 por ser el dato oficial más cercano en fecha al dato de VAB 2008.La clasificación propuesta deriva de un ranking que ubica a los partidos en un continuo de mayor a menor PBG per cápita y divide los 24 municipios del conurbano Bonaerense en tres categorías: alto (20.000 a 50.000 pesos), medio (15.000 a 19.999 pesos) y bajo (6.700 a 14.999 pesos).

2 El indicador de Necesidades Básicas Insatisfechas (NBI) mide pobreza estructural y considera NBI1: Vivienda de tipo inconveniente (vivienda de inquilinato, precaria u otro tipo); NBI2: Carencias sanitarias (sin retrete); NBI3: Condiciones de Hacinamiento; NBI4: Inasistencia escolar; y NBI5: Capacidad de Subsistencia.

${ }^{3}$ A fines de 2015, la coalición Cambiemos, conformada por el Radicalismo y el partido Propuesta Republicana (PRO), gana las elecciones nacionales y se impone, entre otros distritos, en la Provincia de Buenos Aires (tradicional bastión político del peronismo, que se presentó con dos expresiones: el Frente para la Victoria-Partido Justicialista y el Frente Renovador).

${ }^{4}$ Ente autárquico, con representación nacional, provincial y municipal. 